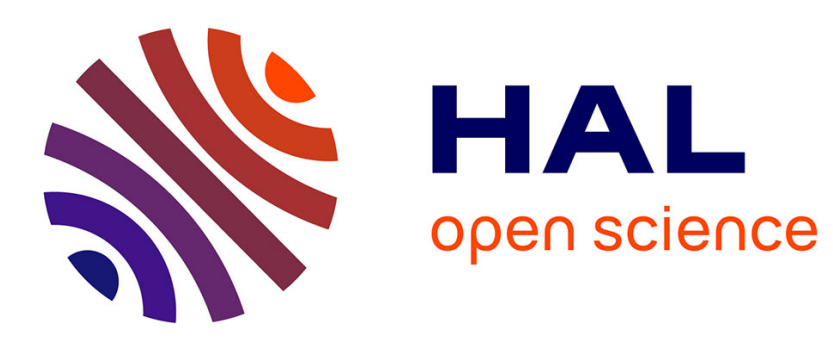

\title{
Régulateur de température pour réfrigérateur à dissolution
}

A. Ghozlan, Daniel Bloyet, E. Varoquaux

\section{To cite this version:}

A. Ghozlan, Daniel Bloyet, E. Varoquaux. Régulateur de température pour réfrigérateur à dissolution. Revue de Physique Appliquée, 1975, 10 (6), pp.463-470. 10.1051/rphysap:01975001006046300 . jpa00243946

\section{HAL Id: jpa-00243946 https://hal.science/jpa-00243946}

Submitted on 1 Jan 1975

HAL is a multi-disciplinary open access archive for the deposit and dissemination of scientific research documents, whether they are published or not. The documents may come from teaching and research institutions in France or abroad, or from public or private research centers.
L'archive ouverte pluridisciplinaire $\mathbf{H A L}$, est destinée au dépôt et à la diffusion de documents scientifiques de niveau recherche, publiés ou non, émanant des établissements d'enseignement et de recherche français ou étrangers, des laboratoires publics ou privés. 


\title{
RÉGULATEUR DE TEMPÉRATURE POUR RÉFRIGÉRATEUR A DISSOLUTION
}

\author{
A. GHOZLAN, D. BLOYET et E. VAROQUAUX \\ Institut d'Electronique Fondamentale (*), Université Paris-Sud, 91405 Orsay, France
}

(Reçu le 29 novembre 1973, révisé le 26 juin 1975, accepté le 30 juin 1975)

\begin{abstract}
Résumé. - Le régulateur de température décrit dans cet article a été conçu pour l'asservissement de la température, à mieux de $100 \mu \mathrm{K}$ près, de systèmes cryogéniques tels que réfrigérateurs à évaporation d'hélium et à dissolution d'hélium-3 dans l'hélium-4. Pour être aisément adaptable aux différentes situations expérimentales, ce régulateur comporte un réseau correcteur avance-retard continuement réglable en fréquence.
\end{abstract}

Abstract. - We describe in this paper a temperature controller designed to maintain the temperature of cryogenic systems such as evaporation or dilution refrigerators constant to better than $100 \mu \mathrm{K}$. This controller can be easily adapted to various experimental situations by means of a continuously tunable lead-lag network.

1. Introduction. - L'exécution de mesures physiques à très basse température, et en premier lieu la mesure de la température elle-même, requiert l'utilisation de dispositifs permettant de maintenir la température à une valeur fixée. Les écarts tolérables de température dans les expériences que nous réalisons à très basse température $[1,2]$ sont inférieurs à une fraction de millikelvin sur des laps de temps de plusieurs heures.

L'objet de cet article est la description du dispositif d'asservissement que nous avons mis au point pour stabiliser la température de différents systèmes : bains d'hélium-4 au voisinage de $1 \mathrm{~K}$, bulbes de mesure de température à hélium-3, chambre de mélange de réfrigérateur à dissolution.

Les dispositifs d'asservissement en usage dans les systèmes cryogéniques sont en général assez rudimentaires car ils doivent s'adapter à des situations physiques qui évoluent en raison de la variation rapide des capacités calorifiques et des résistances thermiques avec la température. Lorsque les systèmes physiques à asservir sont simples et les conditions de stabilité peu astreignantes, les dispositifs d'asservissement font simplement appel à un contrôle proportionnel [3]. Lorsque les systèmes deviennent plus complexes ou les exigences de qualité de l'asservissement de température plus

(*) Laboratoire associé au C. N. R. S. sévères il s'avère nécessaire de rajouter un contrôle par avance ou par retard de phase [4] ou même une combinaison des deux.

Le dispositif d'asservissement de température que nous avons réalisé part du signal d'erreur fourni par un pont de résistance à détection synchrone [5]. Il se compose, comme le montre le diagramme par bloc de la figure 1, d'un contrôle proportionnel, d'un réseau correcteur avance-retard de phase et d'un étage de sortie redresseur et limiteur de tension.

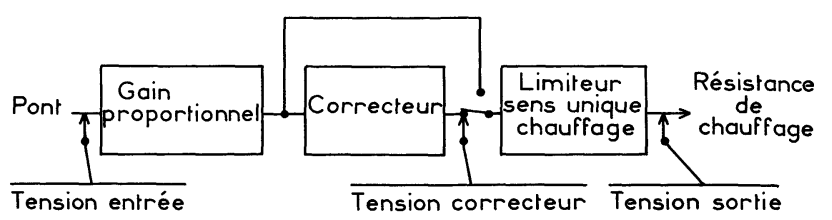

Fig. 1. - Schéma général du régulateur de température.

Le réseau correcteur présente la caractéristique d'être continûment réglable en fréquence. La conception de l'étage de sortie confère au dispositif une grande sûreté de fonctionnement. Dans une version plus complexe, le régulateur était également doté d'un contrôle intégral supplémentaire et d'un extracteur de racine carrée destiné à linéariser la réponse du système pour les signaux d'erreur à grande excursion. Ces deux élé- 
ments ne se sont pas avérés, à l'usage, d'une grande utilité dans le régulateur étudié et n'ont pas été conservés.

Le fonctionnement de ce dispositif d'asservissement a été étudié en le couplant à un système thermique simple. Ce système, opérant au voisinage de la température ambiante, permet de réaliser une maquette simulant les systèmes cryogéniques plus complexes dont il faut réguler la température.

Nous avons ainsi, d'une part observé le comportement réel $\mathrm{du}$ dispositif d'asservissement, d'autre part effectué une analyse théorique de ce comportement par traitement numérique sur ordinateur et par tracé des diagrammes asymptotiques de Bode. Cette étude qui fait l'objet du second paragraphe montre, comme il était attendu, le rôle déterminant du réseau correcteur sur la stabilité du système et permet d'établir une procédure de recherche du réglage optimal. L'établissement préalable d'une telle procédure simplifie la mise en application du régulateur de température aux systèmes cryogéniques en vraie grandeur. Le troisième paragraphe sera consacré à la description des différents circuits composant le dispositif d'asservissement ainsi qu'à l'utilisation du réseau correcteur. Les performances atteintes sur un réfrigérateur à hélium-4 pompé, un bulbe à hélium-3 et un réfrigérateur à dissolution d'hélium-3 dans l'hélium-4 seront exposées en conclusion de cet article.

2. Analyse du fonctionnement du régulateur. 2.1 Introduction. - Pour analyser le fonctionnement du régulateur nous allons étudier son comportement dans l'asservissement de la température d'un système physique simple. Ce système consiste en un réservoir thermique de capacité calorifique $C_{\mathrm{R}}$ porté à une température $T_{\mathrm{R}}$ différente de $T_{\mathrm{A}}$, température de l'environnement ambiant auquel il est couplé par un chemin thermique de résistance $\mathcal{R}_{R}$. La constante thermique de ce réservoir est $\tau_{R}=\mathscr{R}_{R} \mathcal{C}_{R}$. Il reçoit une puissance électrique de chauffage du régulateur, représentée par $V^{2} / R_{\mathrm{c}}$, où $R_{\mathrm{c}}$ désigne la valeur de la résistance de chauffage et $V$ la tension de sortie du régulateur. Sa température est mesurée par un thermomètre dont la constante de temps propre est $\tau_{\mathbf{t}}$. Ce thermomètre est une thermistance dont la résistance est mesurée à l'aide du pont alternatif possédant une constante de temps $\tau_{\mathrm{e}}$, correspondant à un pôle simple.

Un tel système, à trois constantes de temps, représenté schématiquement sur la figure 2, se prête en raison de sa simplicité d'une part, à l'analyse par le calcul, d'autre part, à l'étude expérimentale car il est facile d'en réaliser une maquette dont tous les paramètres sont mesurables et qu'il est possible de perturber d'une manière connue.

La première partie de ce paragraphe est consacrée à l'analyse théorique de l'asservissement en température du système de la figure 2 à l'aide du régulateur schématisé figure 1, fonctionnant sans dispositif correcteur. Nous y établissons les expressions de l'erreur de posi-

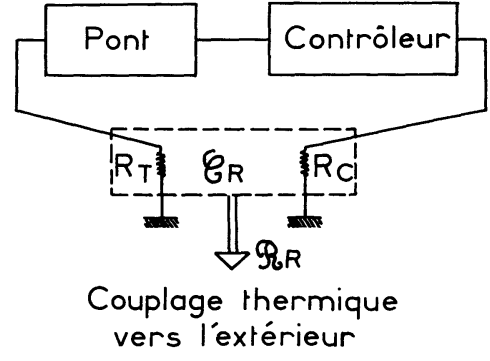

FIG. 2. - Branchement du régulateur : $R_{\mathrm{T}}$ est la résistance thermométrique, $R_{\mathrm{c}}$ la résistance de chauffage, $\mathcal{R}_{\mathrm{R}} \mathrm{C}_{\mathrm{K}}$ la constante de temps thermique principale du système.

tion de l'asservissement, de la raideur de sa réponse à une perturbation ainsi que les conditions de stabilité du système asservi. Dans la deuxième partie nous étudions l'influence du correcteur sur ces conditions de stabilité. Cette étude a été effectuée à l'aide d'un ordinateur à partir des valeurs numériques correspondant au système de la figure 2 . Les résultats obtenus numériquement ont été corroborés par les tracés des diagrammes asymptotiques de Bode. Une comparaison des valeurs expérimentale et théorique de la raideur du système terminera ce paragraphe.

2.2 ANALYSE THÉORIQUE DU SYSTÈME SANS CORRECTEUR. - 2.2.1 Mise en équation du problème.L'évolution des températures du réservoir et de la thermistance $T_{\mathrm{R}}$ et $T_{\mathrm{s}}$ est régie par les équations suivantes :

$$
\begin{gathered}
\mathcal{C}_{\mathrm{R}} \frac{\mathrm{d} T_{\mathrm{R}}}{\mathrm{d} t}+\frac{1}{\mathcal{R}_{\mathrm{R}}}\left(T_{\mathrm{R}}-T_{\mathrm{A}}\right)=\frac{V^{2}}{R_{\mathrm{c}}} \\
\frac{\mathrm{d} T_{\mathrm{s}}}{\mathrm{d} t}=\frac{T_{\mathrm{R}}-T_{\mathrm{s}}}{\tau_{\mathrm{t}}} \\
\frac{V^{2}}{R_{\mathrm{c}}} \simeq \frac{2 \bar{V}_{\mathrm{o}} G_{\mathrm{a}}}{R_{\mathrm{c}}} \frac{\left(\mathcal{C}_{\mathrm{c}}-\mathcal{G}_{\mathrm{s}}\right)}{1+\tau_{\mathrm{e}} p} \text { où } p=j \omega
\end{gathered}
$$

$\mathscr{C}_{\mathrm{s}}(p)$ et $\mathcal{C}_{\mathrm{c}}(p)$ sont les transformées de Laplace des fonctions $T_{\mathrm{s}}(t)$ et $T_{\mathrm{c}}(t), T_{\mathrm{c}}$ désignant la température du point de consigne choisi; $\bar{V}_{0}$ est égal à la valeur moyenne de la tension de chauffage correspondant à la température d'équilibre initiale $T_{\mathrm{s}}(t=0)=T_{\mathrm{s} 0}$. $G_{\mathrm{a}}$ caractérise le gain de la chaîne d'asservissement et est exprimé en $\mathrm{V} / \mathrm{K}$.

Le choix de la condition initiale

$$
\left(\frac{\mathrm{d} T_{\mathrm{s}}}{\mathrm{d} t}\right)_{\mathrm{t}=0}=0
$$

conduit à l'expression suivante de $\mathfrak{C}_{\mathrm{s}}(p)$ :

$$
\begin{aligned}
\mathcal{C}_{\mathrm{s}}(p)=\frac{K T_{\mathrm{c}}}{p\left[K+f(p)\left(1+\tau_{\mathrm{e}} p\right)\right]}+ \\
+\frac{T_{\mathrm{s} 0}\left[\tau_{\mathrm{t}}+\tau_{\mathrm{R}}+p \tau_{\mathrm{t}} \tau_{\mathrm{R}}\right]\left[1+\tau_{\mathrm{e}} p\right]}{K+f(p)\left(1+\tau_{\mathrm{e}} p\right)} \\
+\frac{T_{\mathrm{A}}}{p} \frac{1+\tau_{\mathrm{e}} p}{K+f(p)\left(1+\tau_{\mathrm{e}} p\right)}
\end{aligned}
$$


Dans cette expression $T_{\mathrm{c}}, T_{\mathrm{s} 0}$ et $T_{\mathrm{A}}$ sont des constantes (pour un état d'équilibre donné). $\mathfrak{C}_{\mathrm{s}}(p)$ caractérise la réponse du système se trouvant initialement à la température $T_{\mathrm{so}}$ et soumis à une entrée en échelon ; $K$ est le facteur sans dimension :

$$
K=\frac{2 \bar{V}_{0} G_{a} R_{\mathrm{R}}}{R_{\mathrm{c}}}
$$

$f(p)$ est la fonction $1+p\left(\tau_{\mathrm{t}}+\tau_{\mathrm{R}}\right)+p^{2} \tau_{\mathrm{t}} \tau_{\mathrm{R}} \mathrm{du}$ second degré en $p$.

Par application des théorèmes de la valeur finale et de la valeur initiale [6] il vient :

$$
\begin{aligned}
T_{\mathrm{s}}(t=0) & =\lim _{p \rightarrow \infty}\left[p \mathscr{C}_{\mathrm{s}}(p)\right]=T_{\mathrm{s} 0} \\
T_{\mathrm{s}}(t \rightarrow \infty) & =\lim _{p \rightarrow 0}\left[p \mathscr{C}_{\mathrm{s}}(p)\right]=T_{\mathrm{c}}-\frac{T_{\mathrm{c}}-T_{\mathrm{A}}}{K+1} .
\end{aligned}
$$

Si le premier résultat ne constitue qu'une simple vérification, le second conduit à l'expression de la valeur statique pour les temps longs de l'erreur de position de l'asservissement. Ce résultat, obtenu sans le réseau correcteur, sera également valable pour le système corrigé.

L'évaluation de la réponse du système à une perturbation et de sa raideur s'effectue d'une manière semblable au calcul de l'erreur de position.

Par un circuit auxiliaire, envoyons au temps $t=0$ sur le réservoir de chaleur une puissance thermique perturbatrice $\mathfrak{T}_{\mathrm{p}}$ constante. Cette puissance vient s'ajouter au second membre de l'éq. (1).

La réponse correspondante du système, $\mathcal{C}_{s}(p)$, peut alors être mise sous la forme :

$$
\begin{aligned}
\mathfrak{C}_{\mathrm{s}}(p)=\frac{T_{\mathrm{c}} K}{p\left[K+f(p)\left(1+\tau_{\mathrm{e}} p\right)\right]}+ \\
+\frac{T_{\mathrm{s} 0}\left(\tau_{\mathrm{t}}+\tau_{\mathrm{R}}+p \tau_{\mathrm{t}} \tau_{\mathrm{R}}\right)\left(1+\tau_{\mathrm{e}} p\right)}{K+f(p)\left(1+\tau_{\mathrm{e}} p\right)} \\
+\frac{T_{\mathrm{A}}}{p} \frac{\left(1+\tau_{\mathrm{e}} p\right)}{K+f(p)\left(1+\tau_{\mathrm{e}} p\right)} \\
+\frac{\mathcal{P}_{\mathrm{p}} \mathcal{R}_{\mathrm{R}}}{p} \frac{1+\tau_{\mathrm{e}} p}{K+f(p)\left(1+\tau_{\mathrm{e}} p\right)}
\end{aligned}
$$

L'application du théorème de la valeur finale montre que la température d'équilibre prise par le système perturbé est :

$$
T_{\mathrm{s}}(t \rightarrow \infty)=T_{\mathrm{c}}-\frac{T_{\mathrm{c}}-T_{\mathrm{A}}}{K+1}+\frac{\mathfrak{T}_{\mathrm{p}} \Re_{\mathrm{R}}}{K+1}
$$

Cette relation permet de déterminer la raideur du système asservi, c'est-à-dire l'écart supplémentaire au point de consigne dû à une perturbation donnée.

Cette quantité permet de caractériser de manière simple les qualités en régime d'équilibre de l'asservissement.

Avant d'étudier l'amélioration apportée par le réseau correcteur à la raideur de l'asservissement nous allons considérer les conditions de stabilité de la réponse transitoire du système exprimé par les relations (4) à (8).

2.2.2 Stabilité du système asservi : fréquence d'oscillation et gain critique. - Un système asservi est stable tant que le lieu dans le plan de Nyquist de sa fonction de transfert en boucle ouverte $H_{\text {B.o. n'entoure pas le }}$ point de coordonnées $(-1,0)$ [6].

Afin d'obtenir la fonction du transfert $H_{\text {B.o. }}$ du système que nous considérons, écrivons sa réponse sous la forme :

$$
\mathfrak{G}_{\mathrm{s}}(p)=\frac{K}{1+\tau_{\mathrm{e}} p} \frac{1}{f(p)}\left(\mathcal{G}_{\mathrm{c}}-\mathcal{G}_{\mathrm{s}}\right)+\frac{\mathcal{A}(p)}{f(p)}
$$

forme à laquelle correspond le diagramme de la figure 3. Le terme d'entrée intermédiaire $\mathcal{A}(p)$ est

$$
T_{\mathrm{s} 0}\left(\tau_{\mathrm{t}}+\tau_{\mathrm{R}}+p \tau_{\mathrm{t}} \tau_{\mathrm{R}}\right)+T_{\mathrm{A}}
$$

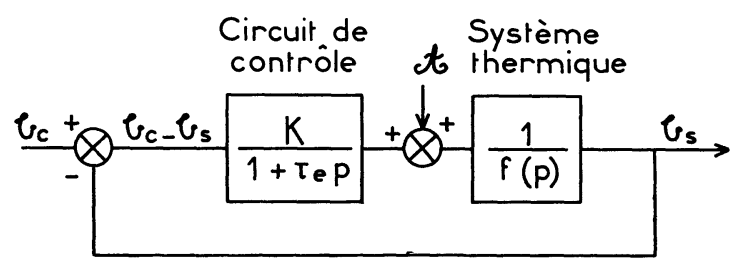

Fig. 3. - Diagramme par bloc de l'asservissement.

Il résulte de la formule (10) que la fonction de transfert en boucle ouverte du système asservi est :

$$
H_{\text {B.o. }}=\frac{K}{1+\tau_{\mathrm{e}} p} \frac{1}{f(p)} .
$$

La connaissance de la fonction de transfert en boucle ouverte $H_{\text {B.o. }}$ livre celle de la pulsation d'oscillation du système bouclé, qui est fixée par le point d'intersection du lieu de $H_{\text {B.o. avec l'axe réel négatif : }}$

$$
\left\{\operatorname{Im}\left[H_{\text {B.o. }}\right]\right\}_{\omega=\omega_{0 s}}=0 \text {. }
$$

Les expressions de la pulsation d'oscillation $\omega_{0 \text { s }}$ la limite d'accrochage et du gain critique correspondant sont les suivantes :

$$
\begin{aligned}
\omega_{0 s} & =\left[\frac{\tau_{t}+\tau_{e}+\tau_{R}}{\tau_{t} \tau_{e} \tau_{R}}\right]^{1 / 2} \\
K_{c} & =\omega_{0 s}^{2}\left[\tau_{t} \tau_{e}+\tau_{e} \tau_{R}+\tau_{R} \tau_{t}\right]-1 .
\end{aligned}
$$

Nous avons mesuré séparément d'une part, les trois constantes de temps $\tau_{\mathrm{e}}, \tau_{\mathrm{t}}, \tau_{\mathrm{R}}$ et d'autre part, les grandeurs caractéristiques $\omega_{0 s}$ et $K_{c}$; les valeurs ainsi obtenues de $\omega_{0 \mathrm{~s}}$ et $K_{\mathrm{c}}$ ont été comparées à celles qui ont été déduites en remplaçant les constantes de temps par leurs valeurs dans les expressions (13) et (14) :

$$
\begin{array}{lll}
\tau_{\mathrm{c}}=0,12 \mathrm{~s} & \omega_{0 \mathrm{~s}_{\text {mesure }}}=1,06 \mathrm{rd} / \mathrm{s} & \omega_{0 \mathrm{~s}_{\text {calcule }}}=1,27 \mathrm{rd} / \mathrm{s} \\
\tau_{\mathrm{t}}=6 \mathrm{~s} & & \\
\tau_{\mathrm{R}}=35,4 \mathrm{~s} & K_{\mathrm{c}_{\text {mesure }}}=170 & K_{\mathrm{c}_{\text {calcule }}}=352 .
\end{array}
$$


Les différentes incertitudes inhérentes à la détermination expérimentale du gain critique expliquent l'écart trouvé entre les valeurs théorique et expérimentale de cette grandeur.

La valeur de $K_{\mathrm{c}}$ déterminée à partir de la relation (14) fixe la valeur maximale de la raideur du système dans le cas simple étudié ici d'un système à trois constantes de temps. L'amélioration de l'asservissement au-delà de cette valeur nécessite la correction de la fonction de

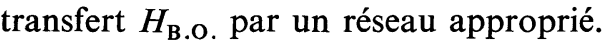

2.3 INFLUENCE DU RÉSEAU CORRECTEUR SUR LA STABILITÉ DU SYSTÈME. - Le système physique réalisé pour illustrer le rôle du réseau correcteur est intéressant à deux points de vue : il a permis d'une part, de développer une analyse théorique du problème, d'autre part, de déterminer expérimentalement les constantes de temps caractéristiques du système. Introduisons maintenant le réseau correcteur, dont nous étudierons ultérieurement l'influence sur la stabilité du système.

2.3.1 Le réseau correcteur. - Le réseau correcteur, que nous venons de mentionner, est un réseau correcteur avance-retard. De tels réseaux sont couramment utilisés dans les problèmes d'asservissement $[7,8]$. Le schéma de bloc de celui que nous avons réalisé est représenté sur la figure 4 . La fonction de transfert correspondante est la suivante :

$$
\mathcal{C}(p) \simeq \frac{\left(p+\omega_{0}\right)^{2}}{\left(p+k \omega_{0}\right)\left(p+\omega_{0} / k\right)} .
$$

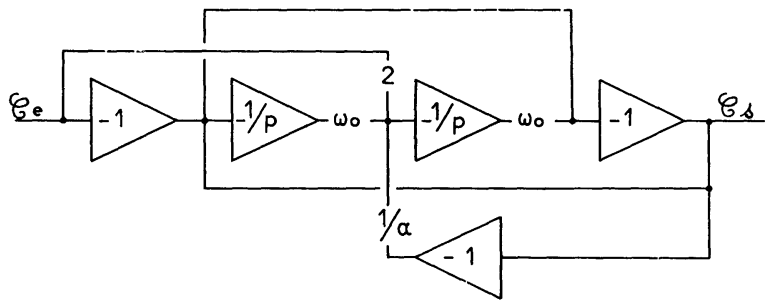

FIG. 4. - Diagramme par bloc du réseau correcteur.

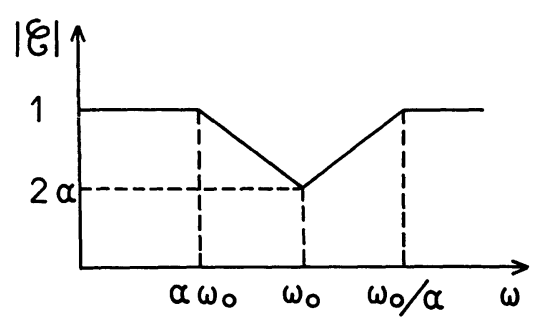

FIG. 5. - Allure de la caractéristique de gain du réseau correcteur.

L'allure de la caractéristique du gain en diagramme de Bode est donnée à la figure 5 .

$\omega_{0}$ désigne la pulsation au voisinage de laquelle on désire faire intervenir le correcteur, dont la zone d'influence est délimitée par les pulsations $k \omega_{0}$ et $\omega_{0} / k$; le facteur $k$ caractérise la profondeur du trou réalisé par le réseau correcteur dans le lieu de la fonction de transfert en boucle ouverte du système $H_{\text {в.o., }}$ c'est-à-dire la réduction de gain introduite par le correcteur (le facteur $k$ est ici choisi inférieur à l'unité). Ce réseau a une double fonction :

- Diminuer le gain de la chaîne d'asservissement au voisinage de la fréquence d'oscillation du système ;

- Introduire une avance de phase qui permet de compenser les déphasages éventuels survenant dans cette région critique.

Nous allons établir les relations qui existent entre le facteur $k$ d'une part, et d'autre part, la réduction de gain et l'avance de phase que le correcteur permet d'obtenir.

Le module de la fonction de transfert (15) peut être mis sous la forme :

$$
|\mathbf{C}| \simeq\left[\frac{\left(\omega_{0}^{2}-\omega^{2}\right)^{2}+4 \omega^{2} \omega_{0}^{2}}{\left(\omega_{0}^{2}-\omega^{2}\right)^{2}+\frac{\omega^{2} \omega_{0}^{2}}{k^{2}}}\right]^{1 / 2}
$$

Il est minimal lorsque la valeur de la pulsation $\omega$ est égale à $\omega_{0}$ et vaut alors :

$$
|\mathbf{C}|_{\omega=\omega_{0}} \simeq 2 k \text {. }
$$

La connaissance de la fonction de transfert $\mathbf{C}(\omega)$ d'un réseau permet de déterminer la phase de ce dernier :

$\Phi(\omega)=2 \operatorname{Arctg} \frac{\omega}{\omega_{0}}-\operatorname{Arctg} \frac{k \omega}{\omega_{0}}-\operatorname{Arctg} \frac{\omega}{k \omega_{0}}(15 \dot{b} \iota s)$

La phase $\Phi$ passe par un maximum pour la valeur suivante de la pulsation

$\frac{\omega_{\max }}{\omega_{0}}=\left[\left(1+k+\frac{1}{k}\right)+\left(1+\frac{1}{k}\right) \sqrt{1+k^{2}}\right]^{1 / 2}$

et la valeur correspondante de la phase est la suivante :

$$
\begin{array}{r}
\Phi_{\max }(\omega) \simeq 2 \operatorname{Arctg} \sqrt{\frac{2}{k}}-\operatorname{Arctg} \frac{1}{k} \sqrt{\frac{2}{k}}- \\
-\operatorname{Arctg} \sqrt{2 k} .
\end{array}
$$

Ces données relatives au réseau correcteur nous permettent d'en étudier l'influence sur la stabilité du système.

2.3.2 Influence du réseau correcteur sur la stabilité du système. - Introduisons la fonction de transfert du réseau $\mathrm{C}(p)$ (15) dans le système d'équations (1-2-3). La réponse du système à un changement de point de consigne peut alors être écrite de la manière suivante :

$$
\tau_{\mathrm{s}}(p)=\frac{K \mathcal{C}(p)}{f(p)\left(1+\tau_{\mathrm{e}} p\right)}\left(\tau_{\mathrm{c}}-\tau_{\mathrm{s}}\right)+\frac{\mathfrak{A}(p)}{f(p)} .
$$

La fonction de transfert en boucle ouverte $H_{\text {B.o.c. }}$ du système corrigé est maintenant définie par la relation :

$$
H_{\text {B.o.c. }}=\frac{K C(p)}{f(p)\left(1+\tau_{\mathrm{e}} p\right)} .
$$


Les expériences ainsi que les calculs numériques ont été effectués en prenant 0,1 pour valeur de $k$.

Le critère de Routh $[6,7]$ a été utilisé pour calculer, à l'aide d'un ordinateur le gain critique du système. Les résultats obtenus dépendent de la position de la pulsation du correcteur $\omega_{0}$ par rapport à la pulsation d'oscillation : la variation correspondante du gain critique est représentée sur la figure 6 .

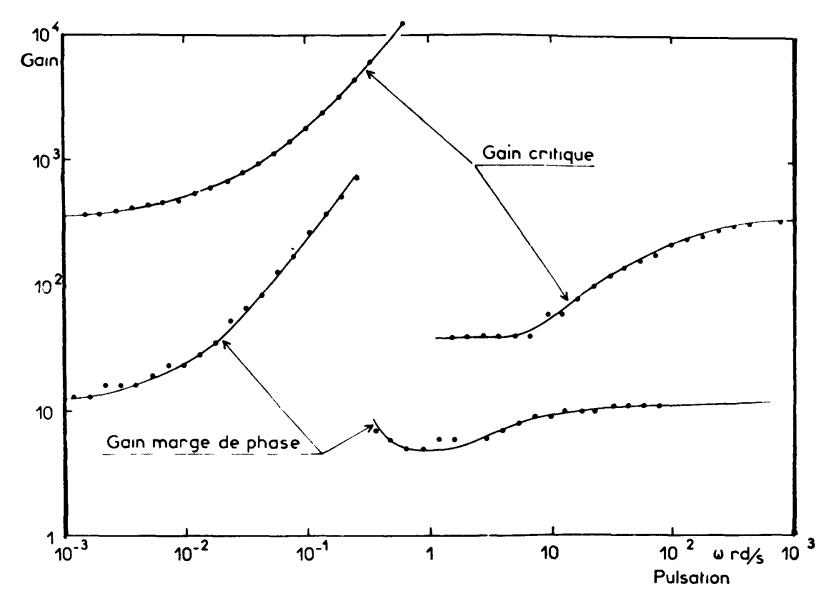

FIG. 6. - Variation du gain critique (courbe supérieure) et du gain maximal compatible avec une marge de phase de $45^{\circ}$ (courbe inférieure) en fonction de la pulsation pour le jeu de valeurs numériques (14).

Le gain critique maximal a pour valeur $12,4 \times 10^{3}$ et il est obtenu pour une valeur de la pulsation du correcteur égale à $0,573 \mathrm{rd} / \mathrm{s}$. Ces résultats corroborent ceux qui ont été déduits du diagramme asymptotique de Bode, pour une valeur donnée de la pulsation du correcteur.

La présence du réseau correcteur augmente donc d'un facteur 35 le gain critique du système, pour les valeurs numériques choisies.

La connaissance du gain critique est insuffisante pour utiliser le dispositif d'asservissement dans les meilleures conditions car la stabilité obtenue en limitant la valeur supérieure du gain au gain critique est conditionnelle. Il est préférable de disposer d'une certaine marge de stabilité qui correspond soit à une marge de gain, soit d'une manière équivalente à une marge de phase.

Nous avons choisi d'assurer au système une marge de phase de $45^{\circ}$ et nous avons évalué le gain maximal $K_{\mathrm{MP}}$ compatible avec cette valeur de la marge de phase.

Ce gain $K_{\mathrm{MP}}$ est déterminé, toujours à l'aide d'un ordinateur, en étudiant la variation $\varphi(\omega)$ de la phase de la fonction de transfert en boucle ouverte du système corrigé $H_{\text {B.o.c. en fonction de la pulsation ; lorsque la }}$ valeur de $\varphi$ est égale à $-135^{\circ}$, la valeur de $K_{\mathrm{MP}}$ est calculée en égalant à 1 le module de $H_{\text {B.o.c. }}$.

Comme pour le gain critique $K_{\mathrm{c}}$, la valeur du gain $K_{\mathrm{MP}}$ dépend de la position de la pulsation du correcteur par rapport à la pulsation d'oscillation. La variation correspondante de $K_{\mathrm{MP}}\left(\omega_{0}\right)$ est représentée sur la figure 6. La valeur maximale de $K_{\mathrm{MP}}$ est égale à 730 et se produit pour une pulsation du correcteur égale à $0,261 \mathrm{rd} / \mathrm{s}$. Remarquons que cette valeur diffère peu de la valeur théorique correspondant à une avance de phase maximale introduite par le correcteur (16). En effet nous avons :

$$
\begin{aligned}
\omega_{0 \mathrm{~s}} & =1,06 \mathrm{rd} / \mathrm{s} \\
\omega_{0} & =0,261 \mathrm{rd} / \mathrm{s} \quad \frac{\omega_{0 \mathrm{~s}}}{\omega_{0}}=4,1 \simeq \sqrt{\frac{2}{k}} . \\
k & =0,1
\end{aligned}
$$

Un réglage convenable du réseau correcteur, sur l'utilisation duquel nous reviendrons au paragraphe 3, permet donc d'augmenter d'un facteur 56 la valeur de $K_{\mathrm{MP}}$, égale à 13 en absence du réseau correcteur.

La raideur du système a été introduite au paragraphe 2.2.2 et définie en fonction du facteur $K=2 \bar{V}_{0} G_{\mathrm{a}} \mathcal{R}_{\mathrm{R}} / R_{\mathrm{c}}$. Une première détermination de la raideur consiste à évaluer les différents facteurs intervenant dans l'expression de $K$. Une seconde détermination peut être effectuée à partir de la définition physique de la raideur, c'est-à-dire l'aptitude du système à minimiser l'écart de la température $T_{\mathrm{s}}$ par rapport à la température du point de consigne, sous l'influence d'une perturbation extérieure :

$$
\rho=\Delta T_{\mathrm{sA}} / \Delta T_{\mathrm{A}}
$$

où les deux écarts $\Delta T_{\mathrm{sA}}$ et $\Delta T_{\mathrm{A}}$ sont respectivement mesurés en l'absence et en présence du système d'asservissement. Nous allons montrer que les résultats de ces deux déterminations sont concordants.

๔) Première évaluation de la raideur. - Les conditions expérimentales dans lesquelles l'influence de la perturbation a été observée, sont les suivantes :

$$
\begin{aligned}
& \bar{V}_{0}=1,65 \mathrm{~V}, \quad G_{\mathrm{a}}=135 \mathrm{~V} / \mathrm{K}, \quad \mathcal{R}_{\mathrm{R}}=111 \mathrm{~K} / \mathrm{W} \\
& R_{\mathrm{c}}=150 \Omega .
\end{aligned}
$$

La valeur du coefficient de raideur ainsi obtenue est $: \rho=320$.

B) Seconde évaluation de la raideur. - Les écarts de température $\Delta T_{\mathrm{sA}}$ et $\Delta T_{\mathrm{A}}$ sont directement mesurés dans les mêmes conditions que précédemment et conduisent aux résultats suivants :

$$
\begin{aligned}
\Delta T_{\mathrm{sA}} & =1,85 \mathrm{~K}, \quad \Delta T_{\mathrm{A}}=6,38 \mathrm{mK} \\
\rho & =290
\end{aligned}
$$

Ces deux déterminations concordent aux erreurs expérimentales près.

3. Description des schémas. - 3.1 L'ÉTAGE D'ENTRÉE ET LE RÉSEAU CORRECTEUR. - Nous allons maintenant décrire les différents schémas du montage électronique réalisé.

Le premier étage, un amplificateur à gain variable représenté sur la figure 7 , fait directement suite au détecteur synchrone avec une seule constante de temps de filtrage, située dans le pont de mesure [5]. La stabi- 


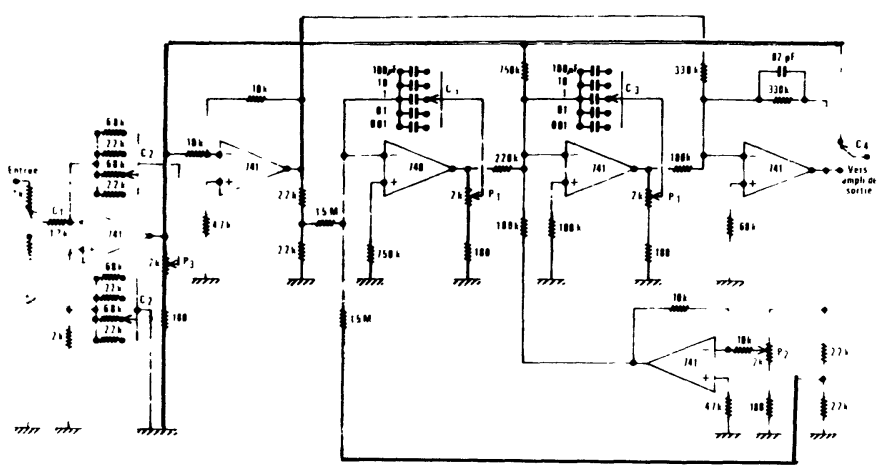

FIG. 7. - Etage d'entrée et correcteur: $\mathrm{C}_{1}$, commutateur d'entrée, $C_{2}^{\prime}, C_{2}^{\prime \prime}$ et $P_{3}$, réglages du gain proportionnel, $P_{1}^{\prime}, P_{1}^{\prime \prime}$, $C_{3}^{\prime}$ et $C_{3}^{\prime \prime}$ réglages de la fréquence du correcteur, $P_{2}$, ajustement de la profondeur du trou, $\mathrm{C}_{4}$, commutateur du correcteur.

lité en température de la tension de décalage de ces deux étages conditionne la stabilité de l'ensemble du dispositif d'asservissement. Elle est meilleure que $50 \mu \mathrm{V} / \mathrm{K}$ dans le montage actuel. Cet amplificateur est suivi du réseau correcteur (illustré par la figure 8 , et également sur la figure 7) dont nous allons maintenant détailler la réalisation et l'utilisation.

3.1.1 Réalisation du réseau correcteur. - Le choix du réseau correcteur a été dicté par la nécessité de disposer de deux paramètres continûment variables : la pulsation du réglage $\omega_{0}$ et le facteur de réduction de gain $k$. Cette maniabilité confère une certaine originalité au dispositif que nous avons réalisé. Le schéma de bloc du réseau correcteur est représenté sur la figure 4 ; la relation obtenue entre les tensions d'entrée et de sortie de ce réseau est la suivante :

$$
\mathrm{C}_{\mathrm{s}}=\mathrm{C}_{\mathrm{e}}\left[1+\frac{2 \omega_{0}}{p}+\frac{\omega_{0}^{2}}{p^{2}}\right]-\mathcal{C}_{\mathrm{s}}\left[\frac{\omega_{0}}{k p}+\frac{\omega_{0}^{2}}{p^{2}}\right]
$$

La fonction de transfert correspondante est une approximation de la fonction de transfert théorique (15), dans l'expression de laquelle le terme $k \omega_{0} / p$ est négligé devant $\omega_{0} / k p$, ce qui est tout à fait licite puisque $k$ est très inférieur à l'unité.
Le montage comprend essentiellement des amplificateurs opérationnels fonctionnant soit en intégrateurs (multiplicateurs par $1 / p$ ) soit en inverseurs.

La constante multiplicative $\omega_{0}$ est obtenue à partir de la pulsation de coupure $\omega_{\mathrm{c}}$ des deux amplificateurs intégrateurs ; un contacteur double à quatre positions permet de modifier la valeur des capacités, c'est-à-dire $\omega_{\mathrm{c}}$; un diviseur potentiométrique, placé à la sortie de chacun de ces amplificateurs fournit une plage de variation de $\omega_{\mathrm{c}}$, comprise entre $0,1 \omega_{\mathrm{c}}$ et $\omega_{\mathrm{c}}$, pour une valeur donnée de $\omega_{\mathrm{c}}$. La constante multiplicative variable $1 / k$ est obtenue en plaçant un diviseur potentiométrique à l'entrée de l'amplificateur inverseur correspondant.

3.1.2 Utilisation du réseau correcteur. - L'utilisation du réseau correcteur peut être aisément déduite des résultats obtenus au paragraphe 2.3.1. En effet l'avance de phase introduite par le réseau correcteur est maximale lorsque la pulsation $\omega$ prend la valeur suivante $: \omega \simeq \omega_{0} \sqrt{2 / k}$. Donc pour stabiliser le système en présence d'une oscillation de pulsation $\omega_{0 \mathrm{~s}}$ il suffira de régler la pulsation du correcteur de manière à satisfaire à la condition suivante :

$$
\omega_{0 \mathrm{~s}} \simeq \omega_{0} \sqrt{\frac{2}{k}}
$$

3.2 L'ÉTAGE DE SORTIE. - L'étage de sortie est représenté sur la figure 8 et possède essentiellement trois fonctions. La première est d'imposer un signe (positif dans le cas du schéma) à la tension de sortie, ceci afin de ne pas chauffer le système lorsque sa température est supérieure à la température à laquelle on désire l'asservir. Deux diodes 1 N 914 situées dans la boucle de contre-réaction d'un amplificateur opérationnel permettent de réaliser cette opération. La seconde fonction est de limiter la tension de chauffage délivrée par le dispositif d'asservissement à une certaine valeur suivant le système physique étudié. On utilise, à ces fins, la courbe de saturation de l'amplificateur de sortie ainsi qu'un diviseur potentiométrique. $\mathrm{La}$ troisième fonction est de fournir une puissance de chauffage au système que l'on désire asservir ; cette

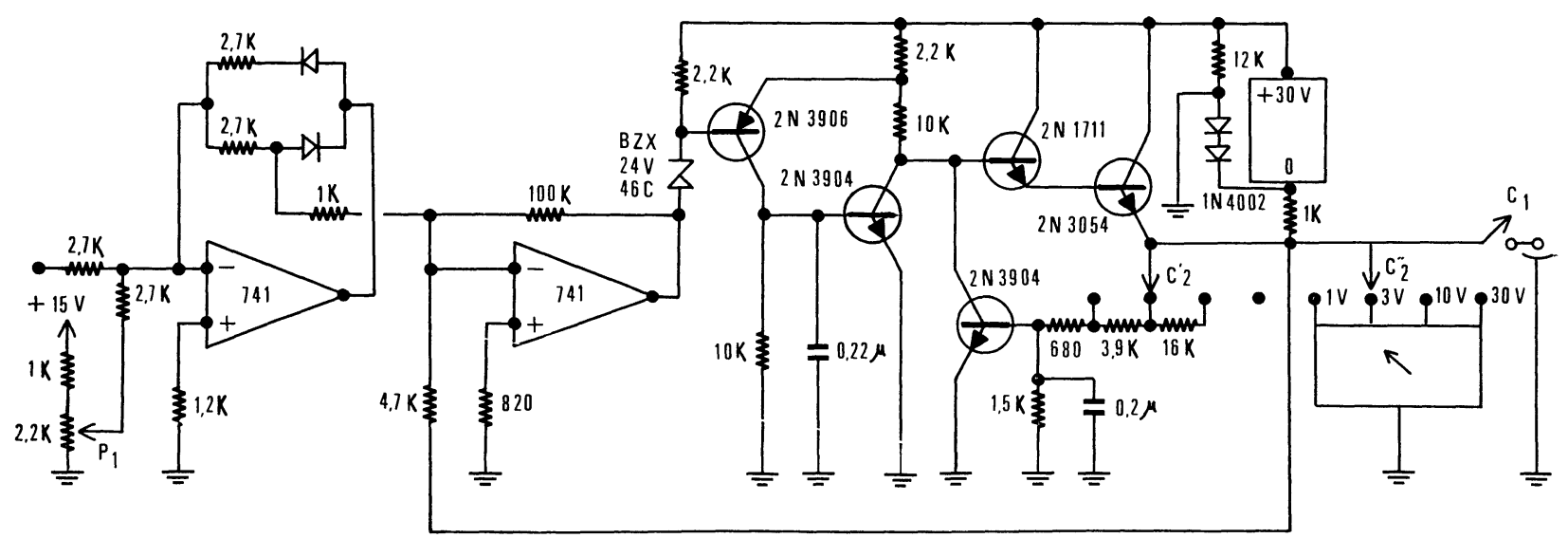

Fig. 8. - Amplificateur de sortie, limiteur et sens unique, $\mathrm{C}_{1}$, commutateur de sortie, $\mathbf{P}_{1}$ réglage de la tension de décalage de zéro, $C_{2}^{\prime}, C_{2}$ commutateurs jumelés de seuil du limiteur et de sensibilité du galvanomètre de sortie. 
dernière est relativement faible (inférieure au watt) et la valeur maximale de la tension de sortie est de $30 \mathrm{~V}$.

4. Conclusion. - Nous venons de montrer, par l'étude théorique et expérimentale d'un système physique simple, que la présence du réseau correcteur améliorait la stabilité du système dont on désirait asservir la température. De plus, la facilité de réglage du réseau correcteur rend le dispositif d'asservissement que nous avons réalisé aisément adaptable aux fonctions de transfert correspondant aux différentes situations expérimentales. Nous l'avons utilisé aux fins d'asservir la température des systèmes cryogéniques suivants :

- Un bulbe à ${ }^{3} \mathrm{He}$, contenant $1 \mathrm{~cm}^{3}$ de ${ }^{3} \mathrm{He}$ et servant à l'étalonnage de résistances. Ce bulbe est connecté par une impédance thermique au distillateur d'un réfrigérateur à dissolution de ${ }^{3} \mathrm{He}$ dans ${ }^{4} \mathrm{He}$. La constante de temps thermique correspondante est de l'ordre d'une dizaine de secondes.

- Une chambre expérimentale de ${ }^{4} \mathrm{He}$, contenant $10 \mathrm{~cm}^{3}$ de $\mathrm{He}_{\text {II }}$ reliée thermiquement à un évaporateur de ${ }^{4} \mathrm{He}$. La constante de temps correspondante est de l'ordre de la seconde.

- La chambre de mélange d'un réfrigérateur à dissolution de ${ }^{3} \mathrm{He}$ dans ${ }^{4} \mathrm{He}$ et contenant $10 \mathrm{~cm}^{3}$ de mélange ${ }^{3} \mathrm{He}-\mathrm{He}_{\mathrm{II}}$ en circulation. La constante de temps thermique mise ici en jeu est de l'ordre de la minute.

Les deux premiers systèmes cités sont très proches du système physique étudié comme modèle au second paragraphe. Ils sont tous deux aisés à asservir.

La stabilité de la température du bulbe à ${ }^{3} \mathrm{He}$ a pu être déterminée avec précision par la lecture, sur une jauge de Bourdon en quartz, de la variation de la pression de vapeur de ${ }^{3} \mathrm{He}$. Les résultats que nous avons obtenus sont les suivants: lorsque la valeur de la température du bulbe est voisine de $1,3 \mathrm{~K}$, la stabilité à court terme est de l'ordre de $3 \mu \mathrm{K}$, tandis que la stabilité à long terme (c'est-à-dire sur un intervalle de temps de quelques heures) est de $10 \mu \mathrm{K}$.

Les résultats relatifs à l'asservissement de la température de la chambre à $\mathrm{He}_{\text {II }}$ sont les suivants : la stabilité à court terme est du même ordre que la stabilité à long terme, la valeur correspondante étant de $20 \mu \mathrm{K}$.

Nous pouvons remarquer que la dérive intrinsèque de ces deux systèmes cryogéniques est faible et que la raideur d'asservissement obtenue est élevée. La stabilité résultante est surtout liée à la stabilité des composants de la chaîne d'asservissement et des résistances au carbone servant à effectuer les mesures. Elle ne correspond pas à des perturbations thermiques du système cryogénique lui-même.

Considérons maintenant les performances obtenues sur le réfrigérateur à dissolution de ${ }^{3} \mathrm{He}$ dans ${ }^{4} \mathrm{He}$ [1]. Ce dernier constitue, comme cela a été signalé ailleurs [2], un système intrinsèquement instable et par conséquent difficile à asservir. Nous avons utilisé deux capteurs de température : une jauge de pression osmotique et une résistance au carbone.
L'utilisation de la jauge de pression osmotique est particulièrement avantageuse en raison d'une part, d'un grand découplage par rapport aux fluctuations du champ magnétique, d'autre part d'un temps de réponse très rapide $(10 \mathrm{~ms}$ pour une valeur de la température de la chambre $T_{\mathrm{MC}}$ voisine de $100 \mathrm{mK}$ ). La stabilité sur la jauge elle-même est très grande et la raideur obtenue est de l'ordre de 15000 .

Cette méthode est utilisable lorsque la température de la chambre de mélange $T_{\mathrm{MC}}$ est inférieure à $300 \mathrm{mK}$ car pour des températures excédant cette valeur, la jauge décroche et par conséquent il s'avère impossible d'asservir la pression osmotique régnant au sein de la chambre de mélange. Les résultats relatifs à ce mode d'asservissement sont les suivants : une dérive lente (c'est-à-dire de l'ordre d'une dizaine de minutes) est réduite par l'asservissement à la valeur insignifiante de $7 \times 10^{-8} \mathrm{~K}$, déterminée à partir du coefficient de raideur. Les dérives rapides (sur des intervalles de temps inférieurs à $100 \mathrm{~s}$ ) ne sont que 100 fois moins compensées. Une perturbation, qui sans asservissement donne une variation de $10 \%$ de $T_{\mathrm{MC}}$ autour de $100 \mathrm{mK}$ s'établissant en $23 \mathrm{~min}$, est résorbée par l'asservissement en moins de $3 \mathrm{~min}$ sans que l'écart de $T_{\mathrm{MC}}$ par rapport au point de consigne ne dépasse jamais $10^{-3}$

La figure 9 illustre le retour à l'équilibre de la chambre de mélange du réfrigérateur à une température de l'ordre de $100 \mathrm{mK}$ après :

a) un changement de point de consigne,

b) une perturbation électrique créée par une impulsion radiofréquence d'un spectromètre à résonance magnétique pulsée et correspondant à une énergie de quelques milliers d'ergs.

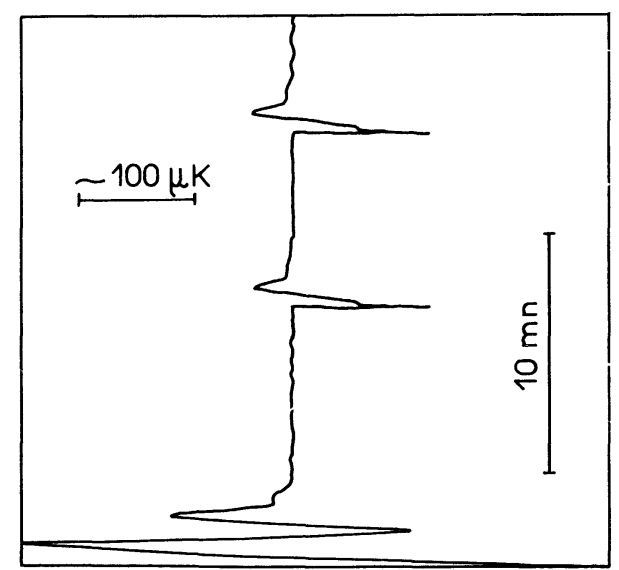

Fig. 9. - Exemple de retour à l'équilibre de la température de la chambre de mélange après perturbation avec réseau correcteur. En l'absence de correcteur le système est sur-critique et ne revient pas à l'équilibre.

Lorsqu'on utilise le second capteur de température, c'est-à-dire la résistance au carbone, le temps de réponse est plus grand (de l'ordre de quelques minutes pour une valeur de $T_{\mathrm{MC}}$ voisine de $100 \mathrm{mK}$ ), la raideur plus faible (voisine de 50) et la stabilité à long terme est 
de $0,4 \mathrm{mK}$. Ces résultats conduisent à penser que les résistances au carbone ne suivent pas exactement la température du liquide à la surface de séparation de phase.

En conclusion l'appareillage électronique qui vient d'être décrit permet d'obtenir des réglages proches de l'optimum dans un grand nombre de situations très diverses; les instabilités de température que nous avons constatées sont liées en général à celles des composants et des thermomètres à haute température et, à plus basse température, au manque de contact thermique.

Ainsi les performances obtenues à l'aide de ce montage sont très satisfaisantes et dépendent surtout des caractéristiques des systèmes dont on désire asservir la température.

\section{Bibliographie}

[1] Chozlan, A., Piéjus, P., Varoquaux, E., C. R. Hebd. Séan. Acad. Sci. 269B (1969) 344.

[2] Bloyet, D., Ghozlan, A., Varoquaux, E., C. R. Hebd. Séan. Acad. Sci. 275B (1972) 375.

Avenel, J., Bloyet, D., Ghozlan, A., Varoquaux, E., C. R. Hebd. Séan. Acad. Sci. 275B (1972) 403.

[3] Sommers, H. S., Rev. Sci. Instrum. 25 (1954) 793.

Larsen, N. T., Rev. Sci. Instrum. 30 (1968) 1.

Röchlin, G. I., Rev. Sci. Instrum. 41 (1970) 73.
Fletcher, R., J. Phys. E : Sci. Instruments 4 (1971) 470.

[4] RIES, R. P. and Moore, B. K., Rev. Sci. Instrum. 41 (1970) 996.

[5] Bloyet, D., Pí́sus, P., Varoquaux, E., à paraître.

[6] Gille, J. C., Decaulne, P., Pellegrin, M., Théorie et calcul des asservissements (Dunod, Paris) 1968.

[7] Di Stephano III, Stubberud, Williams, Feddback and Control Systems (McGraw Hill Book Company) 1967.

[8] MurPHY, Basic Automatic Control Theory (Van Nostrand Company Inc. Princeton, New Jersey) 1957. 